\title{
Use of Rapid Online Data Collection during a Large Community Enteric Outbreak in Toronto, Canada
}

\author{
Anne Arthur, Effie Gournis and Kaitlin Bradley* \\ Communicable Disease Control, Toronto Public Health, Toronto, ON, Canada
}

\section{Objective}

To describe the use of an online survey tool to rapidly collect data from a large community outbreak of enteric illness in Toronto, Canada.

\section{Introduction}

In the early morning of Friday January 20, 2017, Toronto Public Health (TPH) was notified of several reports of acute vomiting, diarrhea, and stomach pain/cramps among students living in residence at a post-secondary institution in Toronto, Canada. A public health investigation was initiated and it was quickly determined that a large number of students and visitors to the campus were affected. Following considerable media coverage, TPH began receiving an overwhelmingly high volume of reports from ill individuals who lived, visited, or worked at the college campus and had experienced gastrointestinal illness.

\section{Methods}

GastroBusters - an established online foodborne illness reporting tool was quickly adapted to support the outbreak investigation. GastroBusters was rapidly updated to include a screening question allowing ill individuals connected with the outbreak location to self-identify and report their symptoms, onset dates and times, and food histories to TPH securely online. The necessary updates were developed, tested, and implemented in less than one hour. Ill individuals were directed to the GastroBusters website - tph. to/gastrobusters - by college administrators and through media messaging. Those who were ill and reported to TPH through other methods (e.g., by phone) were interviewed by TPH investigators to collect comparable data, which were entered by staff into an online survey that mirrored the structure of the GastroBusters questions. These two data sets were merged and descriptive analyses were conducted using MS Excel and SAS v9.2.

\section{Results}

In total, 354 reports associated with the outbreak were received by TPH - 232 who self-reported through GastroBusters, and 122 reported through other methods who were interviewed by TPH. Use of GastroBusters allowed ill individuals to report at a time convenient to them - 204 (88\%) reports were submitted outside of TPH's business hours. As well, by providing ill individuals a method to self-report, TPH was able to rapidly collect, analyze and interpret data over the weekend while minimizing use of TPH staff resources. A summary report was available on Monday January 23, 2017 by 9:00 am, describing 236 confirmed and probable cases whose data were collected via both online surveys (GastroBusters and TPH data collection tool), between Friday and Sunday evenings. These data supported the hypothesis that the source of illness for the outbreak was likely norovirus; this was later confirmed through laboratory results.

\section{Conclusions}

This investigation provides a successful example of how an existing online reporting system for foodborne illness can be used for rapid data collection during a large-scale community enteric outbreak, where the exposed population could not be easily defined and the source of illness was unknown. Advantages of using this approach included: 1) rapid and robust data collection resulting in prompt analysis, and 2) efficient use of public health resources given the volume of reports otherwise processed by a public health investigator. Moreover, the investigation coincided with a weekend when there are fewer staff available and large amounts of overtime costs would have been accrued. TPH is currently developing standards for the use of similar tools in the future.

\section{Keywords}

Foodborne Illnesses; Surveillance; Epidemiology; Infectious Disease Reporting

\section{Acknowledgments}

We would like to acknowledge the leadership and significant contribution of several TPH outbreak leads including: Dr. Michael Finkelstein and Debra Hayden. Additional gratitude is extended to TPH Communicable Disease Control and Health Environments staff, and to our external partners at the college for their cooperation in the investigation of this outbreak. We would also like to acknowledge Sylvia Ota, for providing expertise in the use of GastroBusters during the outbreak.

\section{References}

1. Toronto [Internet]. Toronto: City of Toronto; c1998-2017. GastroBusters; [cited October 2, 2017]. Available from: tph.to/ gastrobusters

*Kaitlin Bradley

E-mail: kaitlin.bradley@toronto.ca 